\title{
Neurocognitive and psychosocial correlates of ventroposterolateral pallidotomy surgery in Parkinson's disease
}

\author{
Henry J. Riordan, Ph.D., Laura A. Flashman, Ph.D., and David W. Roberts, M.D. \\ Department of Psychiatry and Section of Neurosurgery, Dartmouth Medical School, \\ Dartmouth-Hitchcock Medical Center, Lebanon, New Hampshire
}

The purpose of this study was to characterize the neuropsychological and psychosocial profile of patients with Parkinson's disease before and after they underwent unilateral left or right pallidotomy, to assess specific cognitive and personality changes caused by lesioning the globus pallidus, and to predict favorable surgical outcome based on these measures. Eighteen patients underwent comprehensive neuropsychological assessment before and after left-sided pallidotomy (10 patients) or right-sided pallidotomy (eight patients). The findings support the presence of frontosubcortical cognitive dysfunction in all patients at baseline and a specific pattern of cognitive impairment following surgery, with side of lesion being an important predictor of pattern and degree of decline. Specifically, patients who underwent left-sided pallidotomy experienced a mild decline on measures of verbal learning and memory, phonemic and semantic verbal fluency, and cognitive flexibility. Patients who underwent right-sided pallidotomy exhibited a similar decline in verbal learning and cognitive flexibility, as well as a decline in visuospatial construction abilities; however, this group also exhibited enhanced performance on a delayed facial memory measure. Lesioning the globus pallidus may interfere with larger cognitive circuits needed for processing executive information with disruption of the dominant hemisphere circuit, resulting in greater deficits in verbal information processing. The left-sided pallidotomy group also reported fewer symptoms of depression and anxiety following surgery. This enhanced mood functioning was not seen for the right-sided pallidotomy group. No relationships were noted between cognitive impairment or advanced age at baseline and surgical outcome. This study provides objective evidence for specific changes in neurocognitive and psychosocial functioning following left- and right-sided pallidotomy.

\section{Key Words * pallidotomy * neuropsychology * memory * Parkinson's disease}

Stereotactic ventroposterolateral pallidotomy has been shown to be effective in alleviating intractable symptoms of rigidity, bradykinesia, tremor, and medication-induced dyskinesia in patients with advanced Parkinson's disease (PD). This procedure has also been shown to improve gait disturbance and hypophonia in many patients.[16] However, there are very few empirical investigations that have systematically attempted to quantify changes in higher cognitive functioning (such as memory, language, and abstraction) associated with stereotactic ventroposterolateral pallidotomy in patients with PD. There is only one published report that examined neuropsychological variables before and after pallidotomy.[2] This study failed to find any significant differences in 12 patients who underwent pallidotomy (eight on 
the left side and four on the right side) from baseline to their 1-year postoperative follow up. Furthermore, Baron, et al.,[2] did not report any significant difference between patients who underwent right- and left-sided pallidotomy on neuropsychological measures. In addition to investigating the more commonly observed changes in motor functioning, several other preliminary studies have examined alterations in higher cognitive function following pallidotomy. Of these four preliminary reports, two groups $[4,19]$ reported no adverse effects of pallidotomy on higher cognitive functioning, one group[6] reported increased verbal and nonverbal memory, and one group[23] reported mixed results with left-sided pallidotomy patients exhibiting a decline in verbal learning and right-sided pallidotomy patients exhibiting an increase on this measure.

Additionally, there is very limited work that has systematically investigated the effects of pallidotomy on mood functioning or activities of daily living (ADLs). Trepanier, et al.,[23] reported decreased self-reported symptoms of depression following surgery, and Baron, et al.,[2] reported an overall improved quality of life and social functioning for patients who had undergone pallidotomy. The goal of this ongoing prospective study is to characterize the neuropsychological and psychosocial profile of medically intractable patients with PD before and after unilateral left- or right-sided pallidotomy, to assess specific neurocognitive changes caused by lesioning the globus pallidus, and ultimately, to predict favorable surgical outcome from baseline neuropsychological and psychosocial measures.

\section{CLINICAL MATERIAL AND METHODS}

\section{Patient Population}

Our study consisted of 18 patients with advanced PD who were derived from a larger population of 79 patients who had undergone stereotactic pallidotomy at Dartmouth-Hitchcock Medical Center for symptoms of advanced PD between September 1993 and January 1997. Patients with a known psychiatric illness; neurological event, such as cerebrovascular accident, head injury with loss of consciousness; or present or past history of alcohol/substance abuse were excluded from the present study. Patients were included in this study only if they completed a full neuropsychological test battery and measures of psychosocial function before and after surgery. The number of patients returning for follow-up assessment was limited by the distance they had to travel. Of the 23 patients who received complete follow-up evaluations, five were excluded based on the criteria listed above. The remaining 18 patients were then classified into left- and right-sided pallidotomy groups based on their motor symptom profile and handedness. This resulted in two groups: the first group consisted of 10 patients (eight men and two women) who were selected to undergo a unilateral left hemisphere pallidotomy, and the second group consisted of eight patients (four men and four women) selected to undergo a unilateral right hemisphere pallidotomy (chi-square test $=3.39, \mathrm{p}>0.07$ ) The patient groups did not differ in terms of age (left-sided pallidotomy group $=62$, right-sided pallidotomy group $=61.02, \mathrm{p}>0.93$ ), duration of illness (left-sided pallidotomy group $=17.7$ years, right-sided pallidotomy group $=15.4$ years, $\mathrm{p}<0.58$ ) or test-retest interval (left-sided pallidotomy group $=136$ days, right-sided pallidotomy group $=159$ days, $\mathrm{p}>0.05$ ). All patients in the left-sided pallidotomy group were right-handed whereas seven patients in the right-sided pallidotomy group were right-handed and one was left-handed (chi-square test $=2.81, \mathrm{p}$ $>0.24$ ). All patients were taking Sinemet (carbidopa-levodopa) among other anti-PD medications at the time of neuropsychological testing, and all patients reported significant peak-dose dyskinesia and end-dose rigidity. All patients were tested only during "on" periods, the time they were receiving medication, and were evaluated during the same time period within their medication cycle both pre- and postsurgery. All patient underwent a neurological examination as well as comprehensive motor and 
functional testing, including the Simpson Angus Rating Scale and the Activities of Daily Living Scales.

\section{Neurosurgical Procedure}

All surgical procedures were performed using the Leksell G-frame (Elekta Instruments, Atlanta, GA), which was affixed to the patient's head in conventional fashion using a local anesthetic. Computerized tomography scans with 1.5-mm contiguous, nonoverlapping slices (GE 9800; General Electric Medical Systems, Milwaukee, WI) and magnetic resonance images with three-dimensional spoiled gradient 1.5-mm slices (Signa 1.5-tesla scanner; General Electric Medical Systems) were obtained for stereotactic coordinate generation. Initial coordinates were chosen at 18 to $22 \mathrm{~mm}$ lateral to midline, $2.5 \mathrm{~mm}$ anterior to the midcommissural point, and $6 \mathrm{~mm}$ inferior to the commissural plane. The target was then simulated on reformatted magnetic resonance imaging data, and the coordinates were adjusted to optimize its location to within the medial segment of the globus pallidus.

In the operating room, the patient was positioned supine, with the frame secured using a Mayfield adapter. The frontal scalp was shaved, prepared, and anesthetized using 1\% lidocaine with 1:200,000

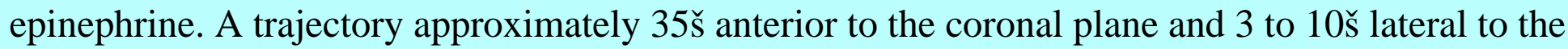
parasagittal plane was chosen. Through a 4-mm skin incision and with the use of the frame bushings as a guide, a twist-drill craniotomy was made using a 3/16-in bit and air-powered drill. The dura was coagulated by electrocautery, and a radiofrequency needle (Radionics, Inc., Burlington, MA) with 1.8-mm outer diameter and 2-mm exposed tip was advanced to the target point.

The patient was continually monitored neurologically throughout the procedure, with particular attention to strength, speech, and visual field function. Physiological confirmation of the target point was obtained using macrostimulation at 5 and $75 \mathrm{~Hz}$ to assure appropriate thresholds for motor and visual response. If this was satisfactory, a temporary lesion was made at 45 š for 60 seconds, with continual assessment of strength, motor, and visual field function. If this too confirmed the location to be satisfactory, a permanent lesion was then made at 75 š for 90 seconds while the patient underwent similar neurological assessment. The probe was then withdrawn $2 \mathrm{~mm}$, and identical physiological testing followed by temporary and then permanent lesioning was performed. A total of five sequential lesions were made in this manner. The probe was then withdrawn, the scalp was sutured with No. 4-0 Prolene suture, and the frame was removed. The patient was observed in the recovery room for 1 hour, a follow-up magnetic resonance image obtained later that evening, and the patient was observed overnight on the neurosurgery ward. Nearly all patients were discharged the following morning.

\section{Neuropsychological Assessment}

A comprehensive battery of cognitive and motor tests designed to assess overall neuropsychological functioning was administered to each patient before and after surgery. The battery took approximately 3.5 hours to administer and included well-established tests that measured intellectual as well as cognitive and motor functioning. All tests were administered by a trained neuropsychology technician or a postdoctoral fellow in neuropsychology.

Neuropsychological summary scales were constructed to encompass eight neuropsychological domains of functioning that were designed to reflect current research and theory on PD. These summary scales were constructed to control for Type I error in the analyses. Dependent measures from the following neuropsychological tests were used to construct the neuropsychological summary scales:

Verbal Reasoning. The verbal reasoning summary scale score was calculated by averaging age-corrected 
scaled scores for the information and vocabulary subtests of the Wechsler Adult Intelligence Scale-Revised (WAIS-R).[24] These tests are designed to assess general and word knowledge and are considered to be relatively insensitive to brain disease.

Verbal Memory. The verbal memory summary scale score was constructed from indices of the of the California Verbal Learning Test (CVLT),[7] including the total number of items recalled achieved for the five trial presentations (in which 16 items are presented aurally over five trials to assess verbal learning and memory), the number of correctly recalled items after a short delay, and the number of correctly recalled items after a 20 minute delay.

Facial Memory. The facial memory summary scale was calculated using the number of correctly identified faces on the Penn Facial Memory Recognition Memory Test[17] in both immediate and delayed conditions. In this test, patients are presented with a group of 20 partially degraded black and white photos of unfamiliar faces and asked to identify those faces from a larger set of 40 faces immediately after studying them and after a 30 minute delay period.

Abstraction. The number of correct categories achieved on a 64-card version of the Wisconsin Card Sort Test (WCST),[11] as well as the number of perseverative errors made during this test were used for construction of this summary scale score. This test measures patients' ability to shift cognitive sets, which is considered an index of executive functioning.

Psychomotor Speed. This summary scale score included the total time to complete Trail Making Test Parts A and B,[16] which measure psychomotor speed, and simple and complex attention and the digit symbol subtest of the WAIS-R.[24] This latter test measures visual motor coordination and response speed, which is relatively independent of intellectual ability.

Language. Four measures of expressive language were used to calculate the language summary scale score. These included the reading recognition score from the Wide Range Achievement Test-III[25] (WRAT-3), which assesses premorbid intellectual functioning and general reading ability; the number of words generated on the Controlled Oral Word Association test,[3] a test of phonemic verbal fluency; the number of animal names[13] that could be generated in 1 minute, a measure of semantic fluency; and the total naming score on the Boston Naming Test,[13] a measure of confrontation naming ability.

Right Hand/Left Hand Motor Assessment. These summary scale scores were derived from a number of motor tasks administered and summary scores were calculated separately for the right and left hands. Tests to assess motor speed and coordination included the Finger Tapping Test of fine motor speed;[16] a fine motor sequencing task;[9] time to completion and number of pegs dropped on the Grooved Pegboard test,[10] a test of manual dexterity and speed; and the Hand Dynamometer Test,[16] a measure of grip strength.

The mean summary scale scores were calculated to allow for easy comparison across neuropsychological and motor functions by providing a common and normalized metric. The use of tests with comparable psychometric properties is important in establishing a differential deficit in any patient population.[5] To calculate these $\mathrm{z}$ scores, each patient's performance was contrasted with normative data and divided by the standard deviation (SD) for that normative data. The z scores within each neuropsychological functional scale were then averaged to achieve a singular summary scale z score, reflecting the average level of performance on a given summary scale. When available, published normative data provided in individual testing manuals were used. When these were not available or were not considered to be age 
appropriate, normative data were taken from other published sources[15,20] and from an unpublished study of 117 healthy elderly control subjects from our laboratory.

Other neuropsychological and motor tests were included in the evaluations but were not used for calculating summary scale scores. These include measures of sensory-perceptual functioning, praxis, mental arithmetic, immediate auditory attention, writing samples, tracing figures, and recognition memory for the CVLT. In addition, patients along with their family members completed a 60-item Activities of Daily Living Questionnaire,[18] designed to assess the impact of cognition and motor dysfunction on daily functioning. Each item was ranked on a 7-point Likert scale with 1 indicating above average ability and 7 being severely disabled. Items were designed to assess functions in one of six domains, including memory, abstraction, attention, language, motor, and mood functioning. This scale provides a subjective self-report analog of patient's objective neuropsychological test performance.

Whenever patients are involved in multiple testing sessions, there is always a concern regarding potential practice effects. In an effort to control for this, alternate test forms or a test interval of greater than 3 months are generally recommended. Whenever possible, alternate forms were used at follow-up (postoperative) assessment. For example, there are well established alternate forms of the CVLT, Trail Making Test, and verbal and semantic fluency tests. This along with the mean test-retest interval of approximately 5 months make it unlikely that any improvement in testing was due solely to practice effects.

\section{Statistical Design and Analysis}

Statistical analyses were conducted using the SAS statistical package (SAS Institute, Cary, NC). As this study generated many dependent measures, neuropsychological and psychosocial summary scales were used whenever possible to control for Type I error. Univariate analyses of variance (ANOVAs) were used to test demographic and pre- and postsurgical neuropsychological data for left-sided and right-sided pallidotomy groups separately. Multivariate analysis of variance (MANOVA) models were used to detect significant differences between left-sided and right-sided pallidotomy groups at baseline for measures of neuropsychological and psychosocial functioning and following surgery in a 2 X 2 X 8 mixed design with left-side/right-side being a between groups variable, and time and domain of functioning being within groups variables. A similar 2 X 2 X 6 design was used for ADL variables. Omnibus MANOVA effects were then decomposed using univariate ANOVA techniques and Bonferroni corrected t-tests. 


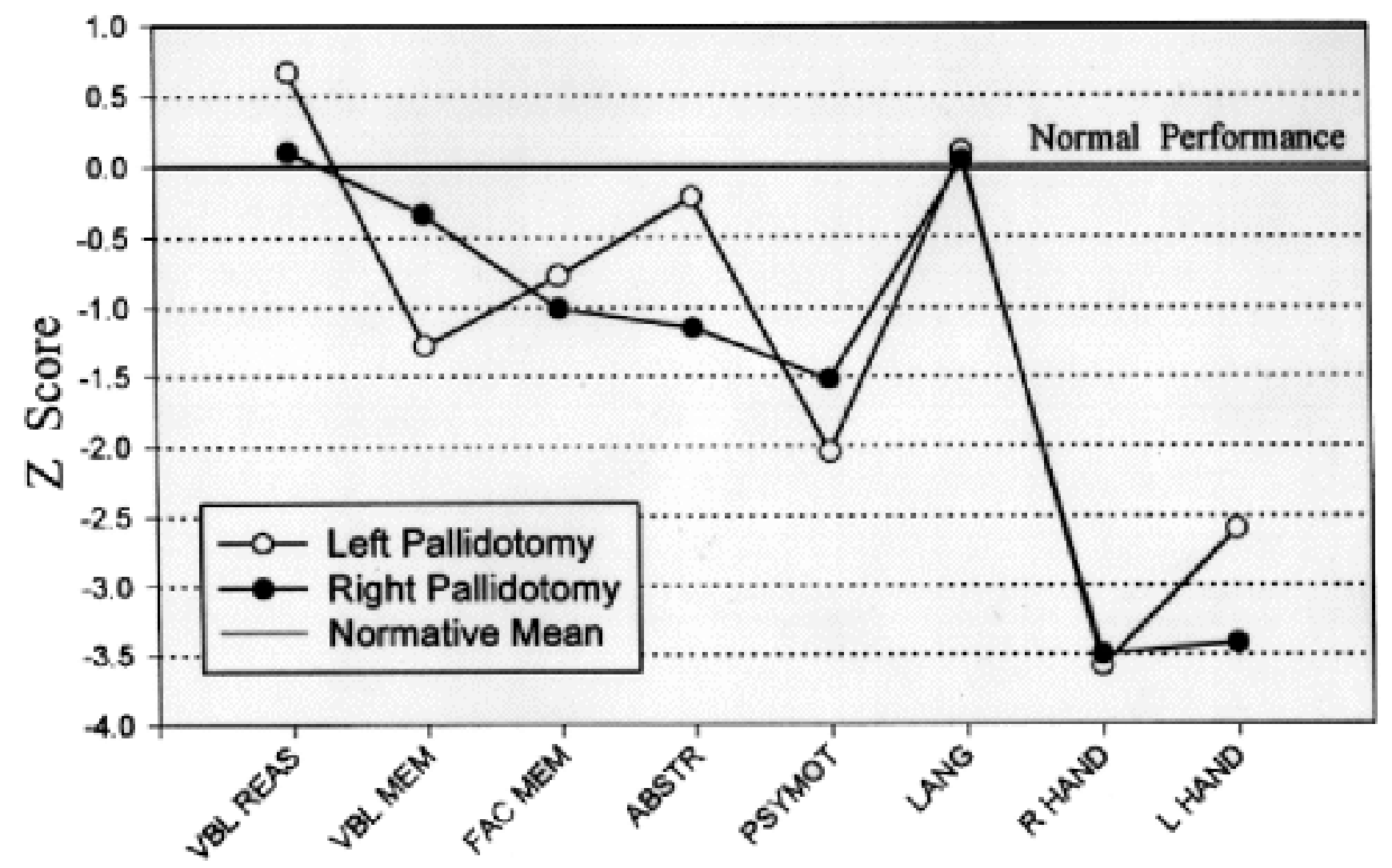

\section{Neuropsychological Summary Scores}

Fig. 1. Graph showing a comparison of 10 left-sided versus eight right-sided pallidotomy patients at baseline on measures of neuropsychological and motor functioning. ABST $=$ abstraction; FAC MEM = facial memory; LANG = language; PSYMOT = psychomotor; VBL MEM = verbal memory; VBL REAS = verbal reasoning.

\section{RESULTS}

\section{Neurocognitive Testing Results:}

A MANOVA using left- versus right-sided pallidotomy group as a between factor yielded a nonsignificant between groups effect $(F[1,15]=0.12, p<0.74)$ suggesting no overall difference between left-sided and right-sided pallidotomy groups across neuropsychological summary scores. However, there was a significant effect for the different neuropsychological summary scores $(F[7]=6.46, p<$ 0.007 ) with both left-sided and right-sided pallidotomy groups performing worse on motor measures. There was no significant interaction between left-sided and right-sided pallidotomy groups and neuropsychological summary scores $(F[7]=2.44, \mathrm{p}<0.11)$. Figure 1 shows for mean neuropsychological summary scores of the left-sided and right-sided pallidotomy groups. 


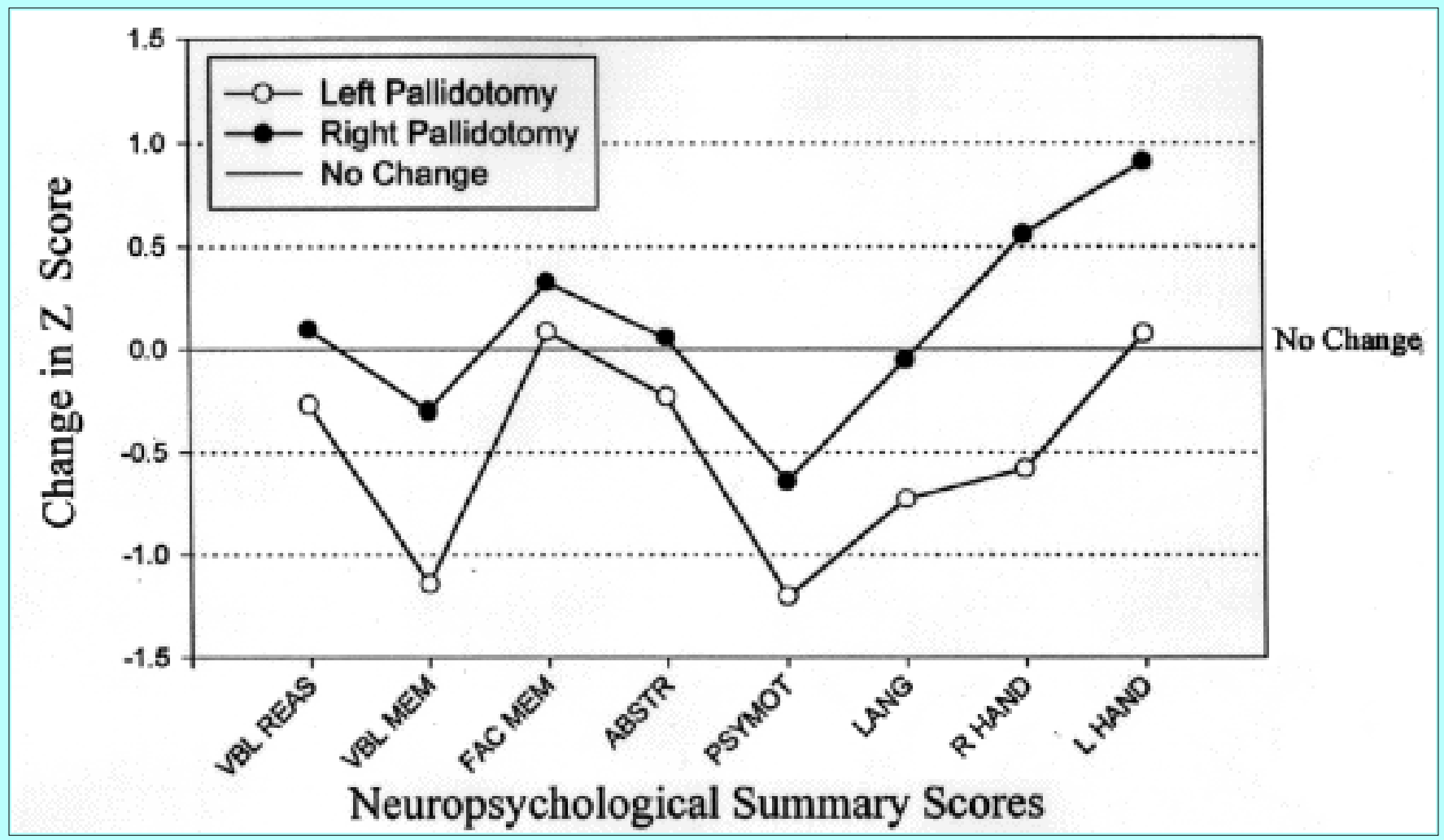

Fig. 2. Graph showing a comparison of 10 left-sided and eight right-sided pallidotomy patients on neuropsychological change scores from preoperative baseline to postoperative follow-up evaluation. ABST = abstraction; FAC MEM = facial memory; LANG = language; PSYMOT = psychomotor; VBL MEM = verbal memory; VBL REAS = verbal reasoning.

Next, a MANOVA using left- versus right-sided pallidotomy group as a between groups factor and change in neuropsychological summary scale scores over time (postsurgical score minus presurgical score) as a within group factor yielded a significant between groups effect $(F[1,14]=11.26, p<0.05)$, a nonsignificant within group effect $(\mathrm{F}[7]=2.03, \mathrm{p}<0.171)$, and a nonsignificant interaction $(\mathrm{F}[7] 0.306$, $\mathrm{p}<0.93$ ). However, significant differences were found between left-sided and right-sided pallidotomy groups on change scores for language and right-hand motor functioning based on Bonferroni corrected $\mathrm{t}$-tests $(\mathrm{p}<0.05)$. Figure 2 shows a comparison of left-sided and right-sided pallidotomy change scores from preoperative to postoperative conditions across neuropsychological summary scale scores. 


\begin{tabular}{|c|c|c|c|c|}
\hline \multicolumn{5}{|c|}{$\begin{array}{c}\text { TABLE } 1 \\
\text { COMPARISON OF NEUROPSYCHOLOGKAL SCORES IN } 10 \text { PATIENTS UNDERGONG } \\
\text { A LEFT-SIDED PALLIDOTOMN }\end{array}$} \\
\hline \multirow[b]{2}{*}{ Test } & \multicolumn{2}{|c|}{ Scores } & \multirow[b]{2}{*}{ F Value } & \multirow[b]{2}{*}{ p Value } \\
\hline & Prestrgical & Postsurgical & & \\
\hline in form ation, 'Wh's-R & 11.4 (2.8) & $10.9(2.1)$ & 2.65 & 0.138 \\
\hline wocabulary whIS-R & $12.6(3.0)$ & $11.5(1.7)$ & 3.12 & 0.111 \\
\hline digit span, W'AlS-R & $9.5(1.7)$ & $9.6(1.3)$ & 0.06 & 0.811 \\
\hline tlock design, Whis-R & $8.1(3.3)$ & $8.0(3.5)$ & 0.02 & 0.879 \\
\hline digit sym bol, 'Whals-R & $7.1(2.1)$ & $7.6(2.9)$ & 0.41 & 0.537 \\
\hline Boston Naming Test & $55.8(3.9)$ & $53.9(7.3)$ & 1.59 & 0.240 \\
\hline 'W'RAT-3 reading & $103.5(11.4)$ & $99.6(12.1)$ & 2.43 & 0.158 \\
\hline phonemic fluency & $32.0(8.6)$ & $27.0(12.6)$ & 5.39 & $0.049 \dagger$ \\
\hline semantic fluency & $20.8(7.5)$ & $14.2(3.8)$ & 10.76 & $0.009 \dagger$ \\
\hline CVLT, total $1-5$ & $36.5(15.9)$ & $24.6(11.2)$ & 9.45 & $0.013 \dagger$ \\
\hline CVLT, short delay & $6.4(3.5)$ & $4.0(2.1)$ & 5.84 & $0.042 \dagger$ \\
\hline CVLT, long delay & $7.0(2.9)$ & $4.6(2.5)$ & 7.36 & $0.026 \dagger$ \\
\hline CVLT, recognition & $13.3(2.3)$ & $14.6(1.8)$ & 1.97 & 0.198 \\
\hline facial memory & $12.7(8.7)$ & $11.6(8.6)$ & 0.14 & 0.715 \\
\hline facial memory, delay & $8.4(7.3)$ & $10.9(6.4)$ & 2.25 & 0.168 \\
\hline WCST categories & $1.6(1.2)$ & $1.6(1.0)$ & 0.00 & 1.00 \\
\hline WCST perse verations & $11.4(4.7)$ & $14.3(6.8)$ & 0.86 & 0.385 \\
\hline Trail Making, Part À & $71.8(68.1)$ & 92.4 (82.4) & 2.30 & 0.173 \\
\hline Trail Making, Part B & $133.7(85.5)$ & $208.2(119.3)$ & 5.85 & 0.060 \\
\hline finger tapping, rt hand & $30.9(10.8)$ & $32.7(13.0)$ & 0.56 & 0.473 \\
\hline finger tapping, It hand & $30.2(10.7)$ & $29.7(14.2)$ & 0.06 & 0.813 \\
\hline finger sequencing, it hand & $7.1(1.6)$ & $7.0(2.9)$ & 0.02 & 0.885 \\
\hline finger sequencing, It hand & $7.3(1.8)$ & $7.0(2.7)$ & 0.40 & 0.541 \\
\hline Grooved Pegboard, it hand & $191.9(78.8)$ & $214.2(82.7)$ & 1.09 & 0.323 \\
\hline Grooved Pegboard, it hand drops & $1.2(0.7)$ & $2.6(3.7)$ & 1.27 & 0.292 \\
\hline Grooved Pegboard, It hand & $184.1(86.9)$ & $176.3(83.1)$ & 0.04 & 0.838 \\
\hline Grooved Pegboard, It hand drops & $0.6(0.5)$ & $1.7(1.2)$ & 4.00 & 0.086 \\
\hline grip strength, rt hand & $32.4(13.1)$ & $28.7(10.6)$ & 6.34 & $0.033 \dagger$ \\
\hline grip strength, It hand & $32.6(14.5)$ & $29.9(11.3)$ & 1.78 & 0.215 \\
\hline
\end{tabular}

An examination of the individual neuropsychological test scores that were used to compose the larger summary scale scores also suggested a unique pattern of change over time for the left-sided and right-sided pallidotomy groups. Tables 1 and 2 outline pre- and postsurgical means and SDs of individual neuropsychological test scores, as well as F and p values. 


\begin{tabular}{|c|c|c|c|c|}
\hline \multicolumn{5}{|c|}{$\begin{array}{c}\text { TABLE } 2 . \\
\text { COMPARISON OF NEUROPSYCHOLOGKAL SCORES IN EIGHT PATIENTS UNDERGONG } \\
\text { RIGHT-SIDED PALLIDOTOW }\end{array}$} \\
\hline \multirow[b]{2}{*}{ Test } & \multicolumn{2}{|c|}{ Scores } & \multirow[b]{2}{*}{ F Value } & \multirow[b]{2}{*}{$p$ Value } \\
\hline & Prestrgical & Postsurgical & & \\
\hline in form ation, 'W'AlS-R & $10.2(1.7)$ & $10.5(2.5)$ & 0.18 & 0.694 \\
\hline wocabulary' 'W'Als-R & $10.4(1.3)$ & $10.7(1.7)$ & 1.34 & 0.285 \\
\hline digit span, 'W'AlS-R & $10.2(1.8)$ & $10.2(2.2)$ & 0.00 & 1.00 \\
\hline block design, Wüs-R & $8.2(1.3)$ & $7.1(1.9)$ & 6.52 & $0.038 \dagger$ \\
\hline digit sym bol, 'MAls-R & $8.4(1.3)$ & $8.7(2.5)$ & 0.21 & 0.662 \\
\hline Boston Naming Test & $55.9(4.0)$ & $55.6(4.5)$ & 0.20 & 0.668 \\
\hline W'RAT-3 reading & $102.5(5.1)$ & $103.5(7.6)$ & 0.44 & 0.526 \\
\hline phonemic fluency & $32.6(10.0)$ & $35.7(9.4)$ & 3.40 & 0.107 \\
\hline semantic fluency & $19.7(3.8)$ & $17.9(2.6)$ & 1.02 & 0.346 \\
\hline CVLT, total $1-5$ & $48.6(8.9)$ & $41.5(8.2)$ & 5.61 & $0.049 \dagger$ \\
\hline CVLT, short delay & $8.4(2.3)$ & $8.6(2.8)$ & 0.10 & 0.756 \\
\hline CVLT, long delay & $9.4(3.6)$ & $8.7(2.4)$ & 0.32 & 0.588 \\
\hline CVLT, recognition & $14.5(1.6)$ & $14.0(1.2)$ & 0.78 & 0.407 \\
\hline facial memory & $11.2(5.0)$ & $11.1(6.9)$ & 0.00 & 0.962 \\
\hline facial memory, delay & $5.7(5.6)$ & $11.5(7.3)$ & 6.97 & $0.033 \dagger$ \\
\hline WCST categories & $1.1(0.8)$ & $1.0(1.3)$ & 0.06 & 0.815 \\
\hline WCST perse werations & $20.6(13.4)$ & $19.1(10.4)$ & 0.59 & 0.468 \\
\hline Trail Making, Part A & $54.7(41.9)$ & $58.4(35.0)$ & 0.49 & 0.506 \\
\hline Trail Making, Part B & $140.0(56.0)$ & $183.1(74.6)$ & 5.70 & $0.048 \dagger$ \\
\hline finger tapping, rt hand & $22.3(8.7)$ & $28.5(11.9)$ & 4.47 & 0.072 \\
\hline finger tapping, It hand & $23.9(9.5)$ & $29.9(9.9)$ & 2.71 & 0.144 \\
\hline finger sequencing, it hand & $6.7(1.2)$ & $7.0(1.1)$ & 1.00 & 0.351 \\
\hline finger sequending, It hand & $6.6(1.6)$ & $6.9(1.2)$ & 1.17 & 0.321 \\
\hline Grooved Pegboard, it hand & $147.4(72.8)$ & $132.1(71.3)$ & 2.75 & 0.141 \\
\hline Grooved Pegboard, it hand drops & $1.7(3.0)$ & $2.1(4.8)$ & 0.28 & 0.611 \\
\hline Grooved Pegboard, It hand & $162.1(52.6)$ & $146.9(68.9)$ & 0.42 & 0.537 \\
\hline Grooved Pegboard, It hand drops & $2.5(2.9)$ & $1.2(1.6)$ & 2.97 & 0.129 \\
\hline grip strength, rt hand & $22.5(10.0)$ & $21.2(8.9)$ & 1.41 & 0.273 \\
\hline grip strength, It hand & $20.9(10.2)$ & $19.9(10.3)$ & 1.92 & 0.215 \\
\hline Data are presented as the & & & & \\
\hline
\end{tabular}

The left-sided pallidotomy group exhibited a significant decline on measures of phonemic and semantic verbal fluency, on total CVLT items, CVLT short and long delay, and right-hand grip strength. The right-sided pallidotomy group also exhibited a significant decline on total CVLT items, as well as a decline on the block design subtest of the WAIS-R and the Trail Making Test Part B. However, the right-sided pallidotomy group exhibited better performance on a delayed condition of the facial memory task from pre- to postsurgical assessments. Although both groups exhibited a significant decline on CVLT total items, a comparison of individual patients' change scores indicated that the patients in the left-sided pallidotomy group exhibited a larger decline on this measure than the right-sided pallidotomy group. Figure 3 details individual patients' change scores from pre- to postsurgical assessment. 


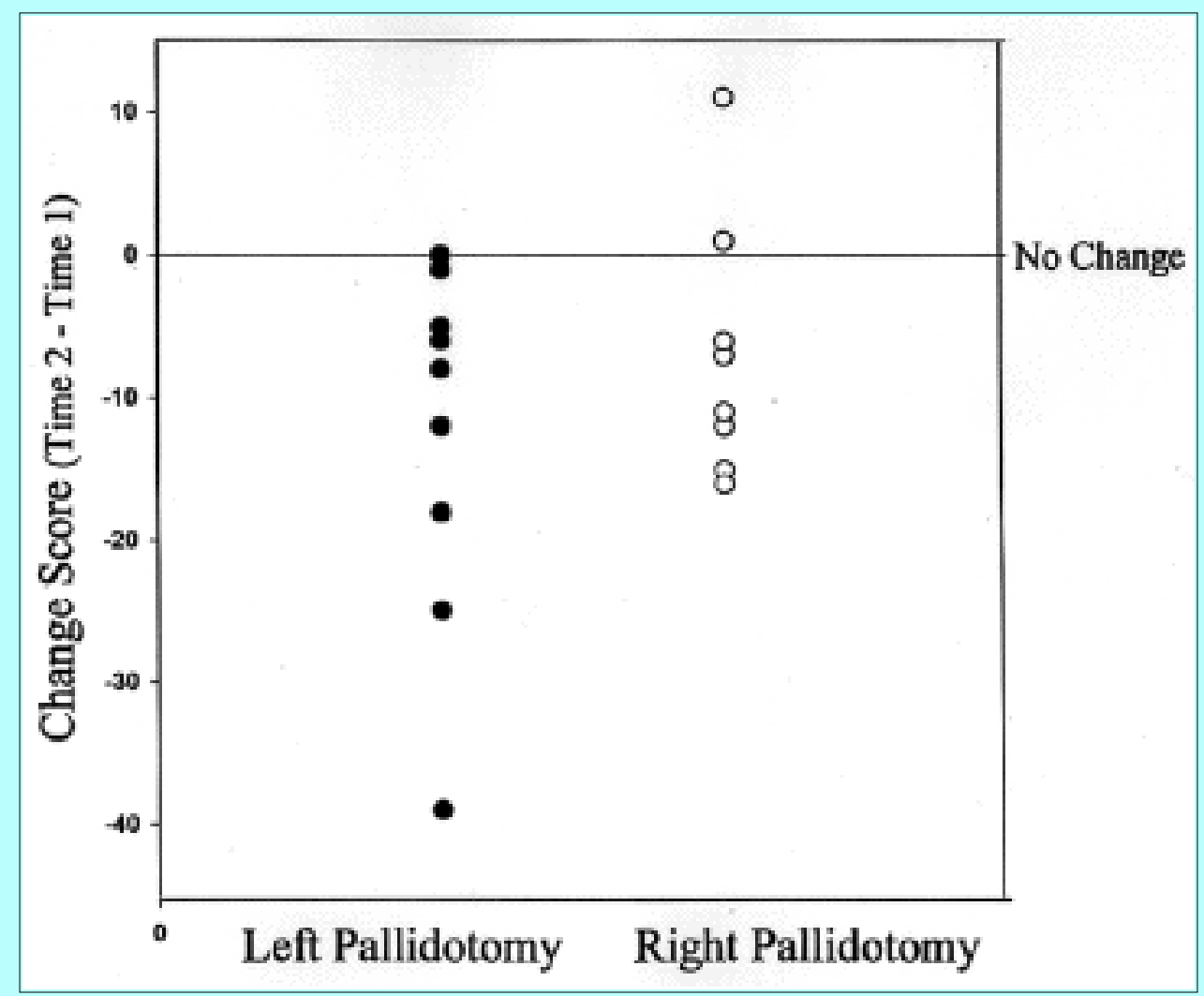

Fig. 3. Graph illustrating individual changes over time in performance on the CVLT for left-sided and right-sided pallidotomy groups.

When the data for all patients were collapsed across surgery type (left vs. right), several significant effects from baseline to postoperative assessment were seen. For example, there was a significant pre-post effect for CVLT total items $(\mathrm{F}=13.8, \mathrm{p}<0.001)$ with performance declining after surgery and improved performance on a delayed facial memory measure $(F=9.38, p<0.007)$ following surgery regardless of side of surgery. 


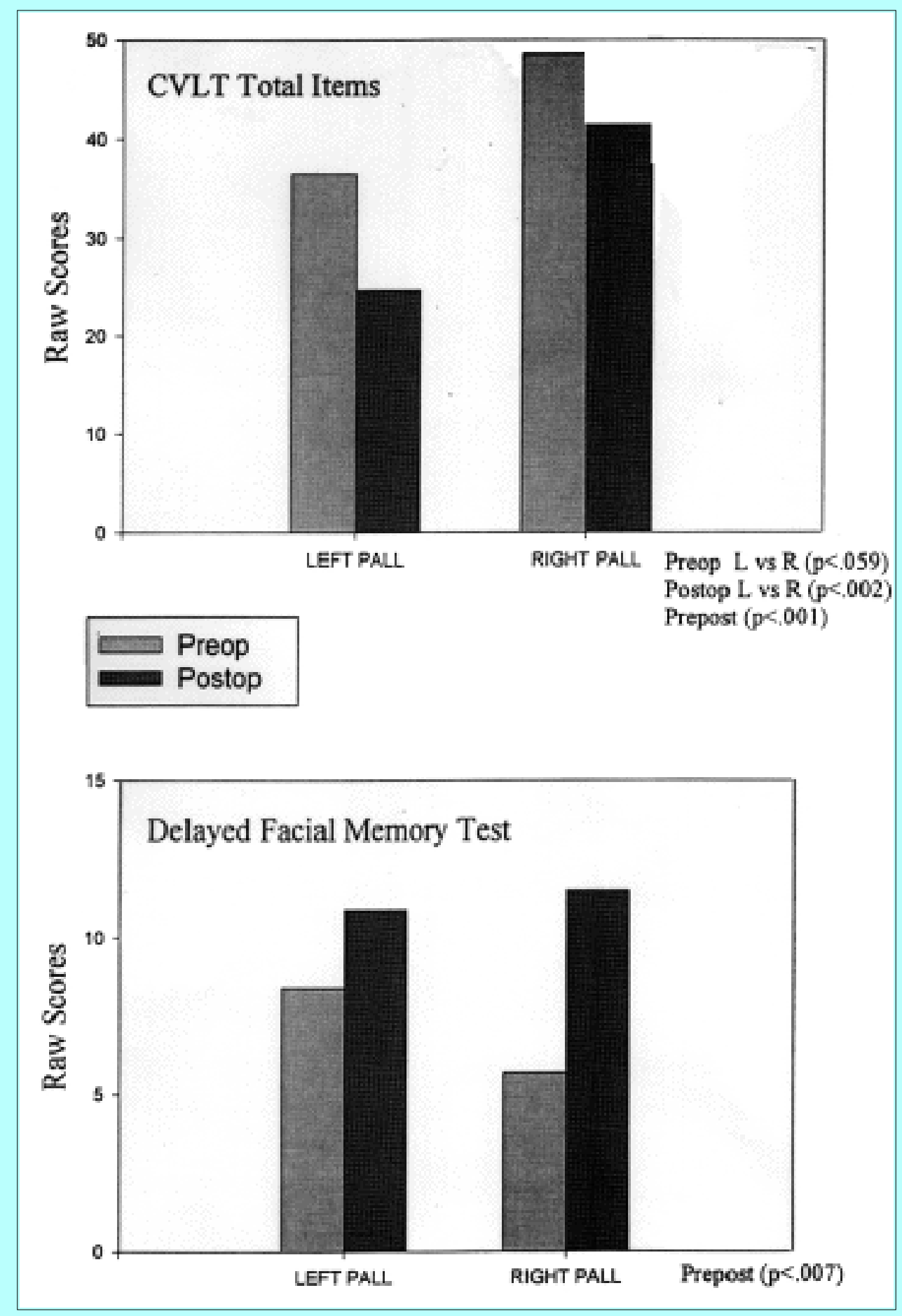

Fig. 4. Bar graphs showing mean memory performance for left-sided (LEFT PALL) and right-sided (RIGHT PALL) pallidotomy groups before and after surgery.

Figure 4 illustrates CVLT and facial memory group means. Similarly, there was a significant pre-post effect for right-hand grip strength $(\mathrm{F}=6.80, \mathrm{p}<0.02)$ with both groups exhibiting weaker grip strength following surgery. This was not seen for left-hand grip strength $(F=0.46, p<0.51)$. Additionally, on measures of fine motor speed, a trend for right-hand finger tapping speed was seen $(F=4.04, p<0.06)$ with all patients exhibiting an increase in tapping speed after surgery regardless of side of surgery. Finally, there was a significant pre-post interaction by side of surgery for number of left-hand drops on the grooved pegboard test of manual dexterity $(\mathrm{F}=6.52, \mathrm{p}<0.02)$ with the right-sided pallidotomy group exhibiting fewer drops after surgery and the left-sided pallidotomy group exhibiting more drops after surgery (Fig. 5). 


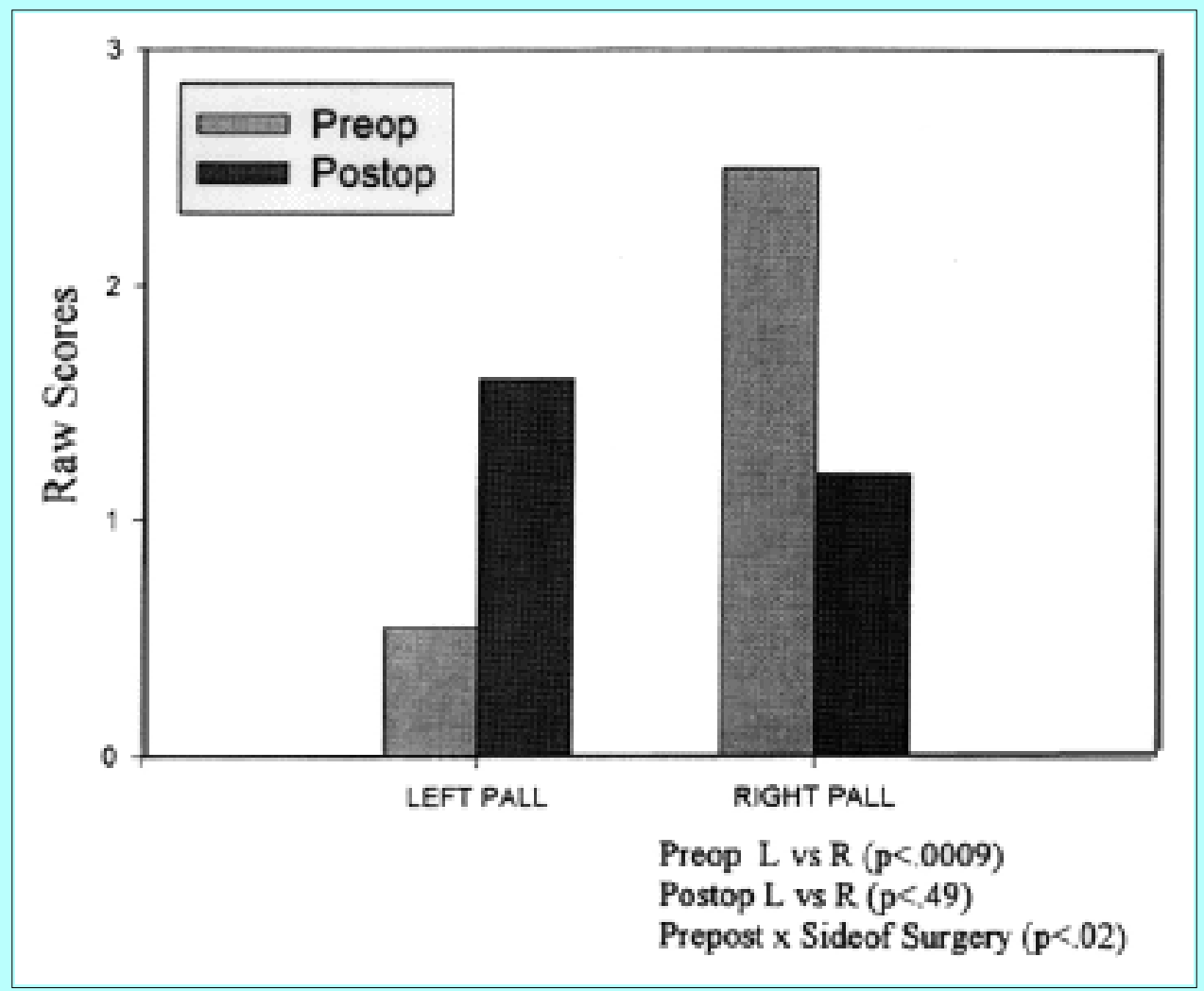

Fig. 5. Bar graph showing mean left-hand drops on the grooved pegboard test for left-sided and right-sided pallidotomy groups before and after surgery.

\section{Psychosocial Results}

A MANOVA using left-sided versus right-sided pallidotomy groups as a between groups factor and ADL functional domains as a within factor failed to yield a significant between groups effect $(F[1,12]=3.48, p$ $<0.09$ ) but suggested a trend for greater improvement on self report measures of functional capacity for the right-sided pallidotomy group. It is important to note that positive changes from pre- to postsurgical assessment indicate worse self-reported functional performance. There was a significant function effect across domains reflecting memory, abstraction, attention, language, motor, and $\operatorname{mood}(\mathrm{F}[5]=2.14, \mathrm{p}<$ $0.17)$. There was no significant interaction between groups and functional domains $(F[5]=0.827, p<$ 0.57). Figure 6 illustrates the mean change scores for left-sided and right-sided pallidotomy groups on the six functional scales. Only the change scores for the functional domain of memory yielded a significant difference with Bonferroni correction ( $\mathrm{p}<0.05$ ), with the right-sided pallidotomy group reporting significant improvement in memory functioning (1 SD) and the left-sided pallidotomy group reporting a very mild decline in memory functioning. When considering all function domains, the right-sided pallidotomy group reported overall increased functional capacity, whereas the left-sided pallidotomy group reported no change or only a very mild decline in functional capacity from preoperative levels. 


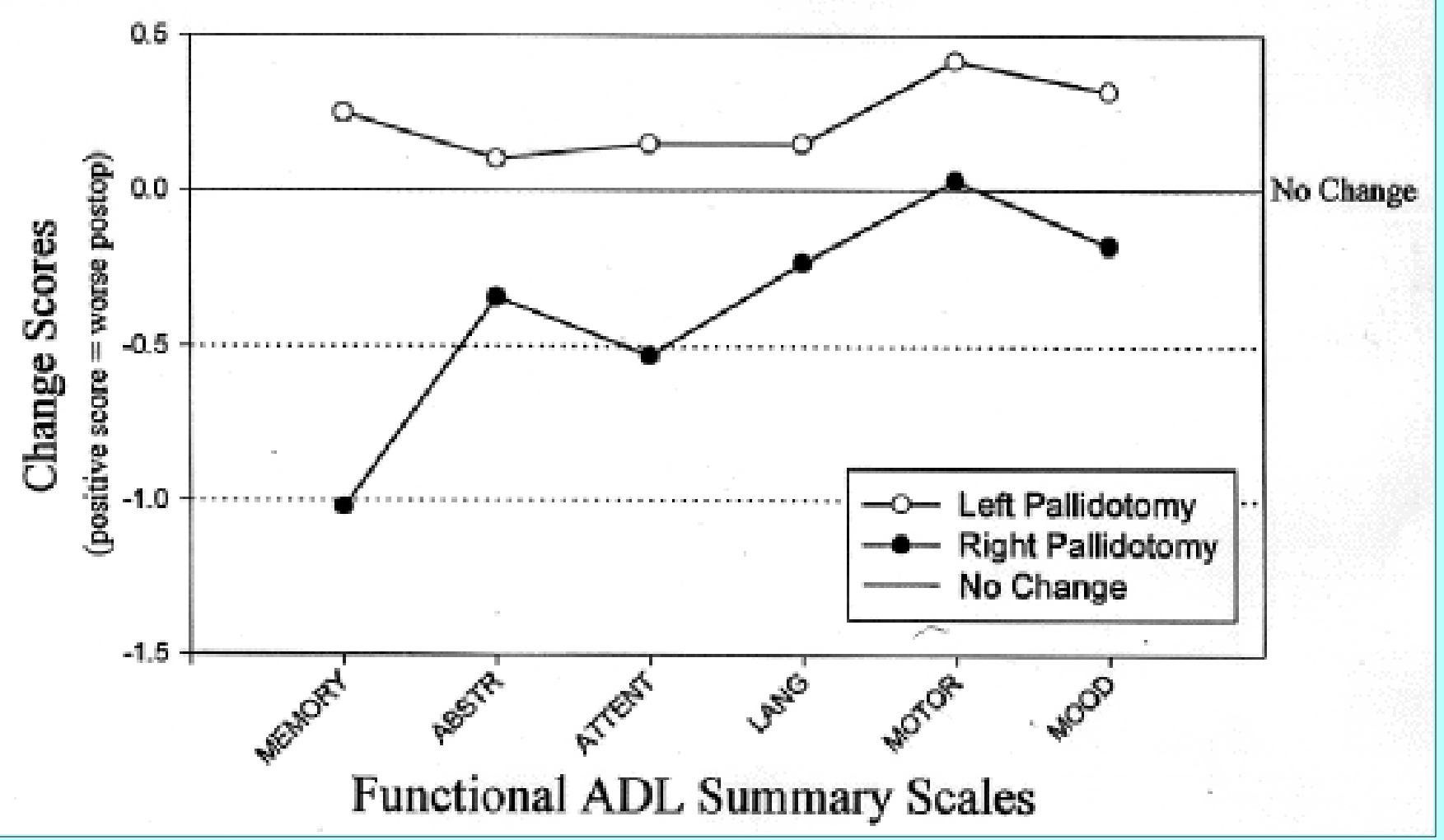

Fig. 6. Graph showing comparison of 10 left-sided and eight right-sided pallidotomy patients on psychosocial change scores from preoperative baseline to postoperative follow-up evaluation. ABST = abstraction; FAC MEM = facial memory; LANG = language; PSYMOT = psychomotor; VBL MEM = verbal memory; VBL REAS = verbal reasoning.

On self-reported measures of depression and anxiety, the left-sided pallidotomy group reported fewer symptoms of depression (left-sided pallidotomy $=10.2$, right-sided pallidotomy $=14.2$ ) and trait anxiety (left-sided pallidotomy $=36.6$, right-sided pallidotomy $=45.5, \mathrm{t}=2.12, \mathrm{p}<0.05$ ) at baseline than the right-sided pallidotomy group. The left-sided pallidotomy group reported even fewer symptoms of depression following surgery whereas the right-sided pallidotomy group continued to report symptoms of mild depression (left-sided pallidotomy $=7.6$, right-sided pallidotomy $=14.6, t=2.1, p<0.05$ ). This resulted in a between groups trend $(\mathrm{F}[1,14]=4.08, \mathrm{p}<0.06$; Fig. 7). As expected, neither group exhibited a significant change in trait anxiety following surgery. Additionally, both patient groups reported similar levels of state anxiety at baseline (left-sided pallidotomy $=45.2$, right-sided pallidotomy $=45.5$ ) and only the left-sided pallidotomy group exhibited a decrease in state anxiety following surgery (left-sided pallidotomy $=35.3$, right-sided pallidotomy $=48.5, \mathrm{t}=2.3, \mathrm{p}<0.05$ ).
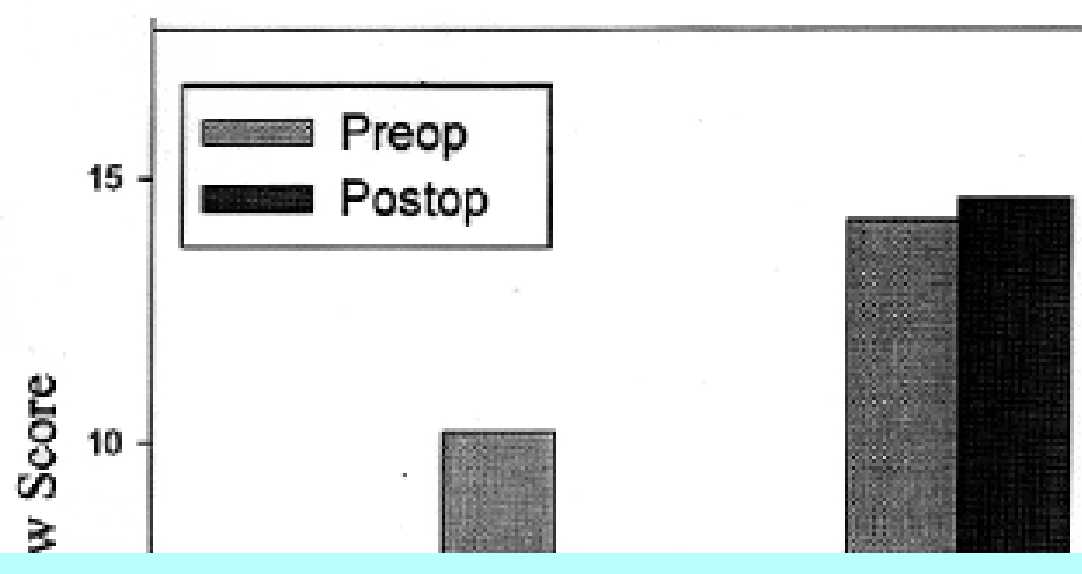

Fig. 7. Bar graph comparing 10 left-sided (LEFT PALL) and eight right-sided (RIGHT PALL) pallidotomy patients on a self-report measure of depression before and after surgery.

\section{Surgical Outcome}

Ratings of rigidity, dyskinesia, 


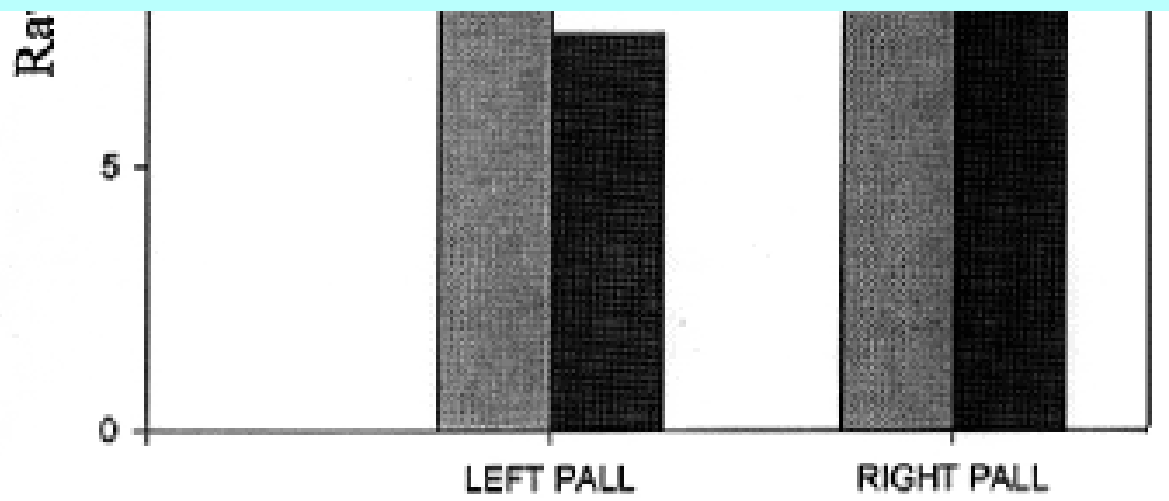

Surgical Side $\mathbf{p}<.05$ bradykinesia, tremor, ambulation, and speech postsurgery were provided by referring neurologists and/or nursing professionals for all patients in this study. These ratings were converted to a common metric reflecting overall surgical outcome with "1" representing substantial improvement in overall functioning, "2" representing some improvement in functioning, "3" representing no discernible change in functioning, and

"4" representing an overall worsening of symptoms. These follow-up ratings were completed an average 8 months postsurgery with no significant difference between groups (left-sided pallidotomy $=7.22$ months, right-sided pallidotomy $=9.28$ months, $t=0.91, p<0.38)$. Fifteen $(83 \%)$ of the 18 patients were rated as having substantial improvement after surgery, and $17(94 \%)$ of the 18 showed at least some improvement. No patient in this study was rated as being worse postsurgery. There was no significant difference between left-sided and right-sided pallidotomy groups on outcome ratings (left-sided pallidotomy $=1.2$, right-sided pallidotomy $=1.25)$, and outcome was not associated with age, overall cognitive status, or affective distress.

\section{DISCUSSION}

This preliminary study suggests a specific pattern of frontosubcortical neuropsychological dysfunction for left-sided and right-sided pallidotomy groups before surgery, with both groups exhibiting a general pattern of motor and psychomotor slowing, decreased grip strength, problems with motor sequencing and coordination, and depression. These deficits along with the relative absence of apraxia, anomia, agnosia, and spared recognition memory (suggesting retrieval rather than consolidation deficits) all support a pattern of subcortical dysfunction, a pattern that has previously been proposed in patients with PD.[8] In addition, there was a specific pattern of neuropsychological and motor deficits for left-sided versus right-sided pallidotomy groups prior to surgical intervention. Specifically, the left-sided pallidotomy group exhibited greater impairment in verbal memory and the right-sided pallidotomy group exhibited greater impairment in facial memory, abstraction, and left-handed motor tasks. This finding supports the use of neuropsychological assessment in lateralizing cognitive and motor impairments, which may prove helpful in making decisions regarding side of initial surgical procedure.

A profile analysis suggests that both right-sided and left-sided pallidotomy groups show a similar pattern of neuropsychological changes following surgery, with the left-sided pallidotomy group exhibiting greater decline across neuropsychological measures. More specifically, patients undergoing left-sided pallidotomy exhibited a mild decline on measures of verbal memory and psychomotor speed. When contrasting individual tests of neuropsychological functioning, the left-sided pallidotomy group exhibited significantly poorer performance on test of verbal fluency (Controlled Oral Word Association and animal naming), verbal learning and memory (CVLT total items, short and long delay, but not recognition) and right-handed grip strength. This is somewhat consistent with reports that the left globus pallidus lesions result in a 2.2 SD decline in verbal learning capacity.[23] Additionally, there was a trend for decreased cognitive flexibility on the Trail Making Test Part B in the left-sided pallidotomy group. All of the tests given are reportedly sensitive to left frontal and temporal system damage. Similarly, patients undergoing right-sided pallidotomy exhibited a significant decline in CVLT total items and the Trail Making Test 
Part B. In addition, the right-sided pallidotomy group also exhibited a significant decrease on the block design subtest of the WAIS-R, a measure of visual spatial constructional abilities. The right-sided pallidotomy group exhibited a significant increase on the delayed condition of the facial memory recognition test (a test with no motor component). Although some of these tests are thought to be sensitive to right hemisphere damage (block design and Trail Making Test B), others such as the CVLT are not.

Lesioning the globus pallidus is thought to have a favorable effect on symptoms of PD by interrupting the subthalamopallidal pathways that result in disinhibition of pallidal activity. It is possible that this procedure also disrupts larger frontosubcortical circuits needed for processing information. Although the motor pathways important in PD are fairly well established,[1] the cognitive circuits important in this disorder have not been well elucidated. Taylor and Saint Cyr[21] proposed a "cognitive loop" that incorporates association cortex, caudate nucleus, globus pallidus/substantia nigra reticulata, ventral lateral and dorsal medial nuclei of the thalamus, and finally the dorsal lateral prefrontal cortex to account for the executive dysfunction often seen in PD. It is possible that damage to any one of the structures (such as that caused by lesioning the globus pallidus) may disrupt the entire cognitive loop causing deficits in attention/concentration, abstraction, psychomotor speed, and memory. In fact, disrupting this circuit may even result in behavioral changes such as disinhibition, apathy, or depression. As our study indicates, it is possible that the adverse effects of disrupting this circuit are more evident when this disruption occurs in the dominant hemisphere, which is essential for verbal information processing. The relative sparing of facial memory for both left-sided and right-sided pallidotomy groups following surgery suggests that facial memory functioning is relatively insensitive to disruption of this circuit. This may be because of its dependence on more posterior structures such as the occipital lobe. The fact that the right-sided pallidotomy group exhibited a significant increase in delayed facial memory recognition following surgery is somewhat unexpected and may reflect a possible rebound of the right hemisphere dopaminergic system that plays a critical role in PD-related cognitive decline.[22]

The subjective functional ADL ratings summary scale was designed to parallel the neuropsychological summary scales used in this study. On these self-report measures it is interesting to note that the left-sided pallidotomy group failed to report any significant changes or reported only very minimal decline across functional domains (such as, memory, abstraction, attention, language, motor, and mood). This is largely consistent with the objective neuropsychological test finding for this group, with the exception of their reported lack of verbal memory problems following surgery and their denial of changes in mood functioning despite endorsement of less depression and anxiety on formal assessment during neuropsychological testing (Beck Depression Inventory and State-Trait Assessment Inventory). The general lack of subjective changes in ADL functional domains for the left-sided pallidotomy group is in contrast to the subjective increase in ADL functioning reported by the right-sided pallidotomy group. Specifically, the right-sided pallidotomy group reported improved ADL functioning on measures of both memory and attention functioning. This group's self report of increased memory functioning is consistent with their objective neuropsychological test finding of better delayed facial memory and lack of short and long delay verbal memory difficulties postsurgery.

Interestingly, despite observed qualitative improvements in motor functioning, such as decreased contralateral rigidity, dyskinesia, bradykinesia, and tremor seen in all patients, no quantitative differences were noted on neuropsychological measures of motor functioning and neither group reported significant improvements in motor functioning as assessed by the ADL scale. Evaluation with these instruments when the patient is in the "on" state may be insensitive to changes caused by lesioning the globus 
pallidus, suggesting a need to develop more sensitive measures.

Finally, concerns have been raised regarding the appropriateness of performing pallidotomy in patients with severe cognitive impairment or dementia. Although the current study size is small, our preliminary results do not indicate any significant relationship between surgical outcome (a composite index of rigidity, dyskinesia, bradykinesia and tremor) and the patient's baseline level of cognitive impairment. In fact, one-third of the patients in this study were determined to have moderate-to-severe global cognitive impairment at baseline. This cognitively impaired group did not differ on objective measures of motor functioning or on overall ratings of neurosurgical outcome. This suggests that cognitively impaired patients may benefit from pallidotomy. Inconsistencies between this preliminary study and other reports regarding changes in neuropsychological functioning following pallidotomy may be partially explained by the use of relatively homogeneous patient samples in other studies that typically excluded patients older than 65 years of age and those patients judged to be cognitively impaired on a brief dementia screening measure. Samples with such exclusions may not accurately reflect the population of patients with PD in general, as $30 \%$ of this population is estimated to have dementia based on mental status examination and Diagnostic and Statistical Manual of Mental Disorders III-Revised criterion.[12]

In sum, this preliminary study suggests specific cognitive impairments before and after pallidotomy with side of surgery being an important predictor of pattern of cognitive impairment. Differences were also noted in self-reported domains of psychosocial functioning following surgery for the left-sided versus right-sided pallidotomy group. It is important to note that the changes demonstrated in neuropsychological function were relatively mild and regularly outweighed by the improvement in the previously intractable motor symptoms of PD. Preliminary analyses also indicate that degree of cognitive impairment is not associated with surgical outcome. However, an increased sample size is needed to characterize clearly the relationship between pallidotomy and neurocognitive functioning before and after surgery.

\section{References}

1. Alexander G, Crutcher M, DeLong D: Basal ganglia-thalamocortical circuits: parallel substrates for motor, oculomotor, prefrontal and limbic functions, in Uylings H, Von Eden C, DeBruin J, et al (eds): The Prefrontal Cortex: Its Structure, Function, and Pathology. Progress in Brain Research, Vol 85. New York: Elsevier, 1990

2. Baron MS, Vitek JL, Bakay RE, et al: Treatment of advanced Parkinson's disease by posterior GPi pallidotomy: 1-year results of a pilot study. Ann Neurol 40:355-366, 1996

3. Benton A, Hamsher K: Multilingual Aphasia Examination, ed 2. Iowa City, IA: University of Iowa, 1989

4. Cahn D, Heit E, Sullivan E, et al: Three-month follow up of posterior ventral pallidotomy. J Int Neuropsychol Soc 3:60, 1997

5. Chapman L, Chapman. J: Strategies for resolving the heterogeneity of schizophrenia and their relatives using cognitive measures. J Abnorm Psychol 98:357-366, 1989

6. Cullum C, Lacritz L, Frol A, et al: Effects of pallidotomy on cognitive function in Parkinson's disease. J Int Neuropsychol Soc 3:61, 1997 
7. Delis DC, Kramer JH, Kaplan E, et al: California Verbal Learning Test. Research Edition. San Antonio, TX: The Psychological Corporation, 1983

8. Dubois B, Boller F, Pillon B, et al: Cognitive deficits in Parkinson's disease, in Boller F, Grafman (eds): Handbook of Neuropsychology. New York: Elsevier, 1991

9. Golden CJ, Hammeke TA, Purisch AD: The Luria-Nebraska Neuropsychological Battery. Los Angeles: Western Psychological Services, 1982

10. Grooved Pegboard Test of Manual Dexterity. Indiana: Lafayette Instrument Co., 1995

11. Heaton R: A Manual for the Wisconsin Card Sorting Test. Odessa, FL: Psychological Assessment Resources, 1981

12. Hughes AJ, Daniel SE, Blankson S, et al: A clinicopathologic study of 100 cases of Parkinson's disease. Arch Neurol 50:140-148, 1993

13. Kaplan E, Goodglass H, Weintraub S: The Boston Naming Test. Philadelphia: Lea \& Febiger, 1983

14. Laitinen LV, Bergenheim AT, Hariz MI: Leksell's posteroventral pallidotomy in the treatment of Parkinson's disease. J Neurosurg 76:53-61, 1992

15. Lezak MD: Neuropsychological Assessment, ed 3. New York: Oxford University Press, 1995

16. Reitan RM, Wolfson D: The Halstead-Reitan Neuropsychological Test Battery: Theory and Clinical Interpretation. Tucson, AZ: Neuropsychology Press, 1985

17. Saykin A, Gur RC, Gur RE, et al: Normative neuropsychological test performance: effects of age, education, gender and ethnicity. Appl Neuropsychol 2:79-88, 1995

18. Saykin A, Robinson L, Stafiniak P., et al: Neuropsychological changes after anterior temporal lobectomy, in Bennett TL (ed): The Neuropsychology of Epilepsy. New York: Plenum, 1992, pp. 263-290

19. Soukup V, Ingram F, Schiess M, et al: Cognitive sequelae of posteroventral pallidotomy: preliminary results. Arch Clin Neuropsychol (In press, 1997)

20. Spreen O, Strauss E: A Compendium of Neuropsychological Tests: Administration, Norms, and Commentary. New York: Oxford University Press, 1991

21. Taylor AE, Saint-Cyr JA: The neuropsychology of Parkinson's disease. Brain Cogn 28:281-296, 1995

22. Tomer R, Levin BE, Weiner WJ: Side of onset of motor symptoms influences cognition in Parkinson's disease. Ann Neurol 34:579-584, 1993

23. Trepanier L, Saint-Cyr A, Lang A, et al: Effects of GPi pallidotomy on neuropsychological function in Parkinson's disease. Arch Clin Neuropsychol. (In press, 1997)

24. Wechsler D: Wechsler Adult Intelligence Scale-Revised. San Antonio, TX: The Psychological Corp., 1981 
25. Wikinson G: Wide Range Achievement Test. 1993 Edition. Delaware: Jastak Associates, 1993

Manuscript received January 27, 1997.

Accepted in final form February 19, 1997.

Address reprint requests to: David W. Roberts, M.D., Section of Neurosurgery, Dartmouth-Hitchcock

Medical Center, One Medical Center Drive, Lebanon, New Hampshire 03756-0001. 